\title{
The role of optical coherence tomography guidance in scaffold versus stent optimization
}

\author{
Arif A. Al Nooryani', Nagwa A. Abdelrahman ${ }^{1,2^{*}}$, Hatem A. Helmy², Yehia T. Kishk² and Ayman K. M. Hassan ${ }^{2}$
}

\begin{abstract}
Background: Optical coherence tomography showed a great ability to identify adverse features during percutaneous coronary intervention with drug-eluting stents and resulted in better clinical outcomes. The study aimed to assess the impact of optical coherence tomography on intraoperative decision-making during implantation of Absorb bioresorbable scaffolds versus everolimus drug-eluting stents.

Results: We performed an observational study that included 223 consecutive patients post optical coherence tomography-guided implantation of either Absorb bioresorbable scaffolds (162 patients) or everolimus drug-eluting stents (61 patients). We studied the influence of optical coherence tomography on intraoperative decision-making during implantation of bioresorbable scaffolds versus drug-eluting stents by analyzing the total rate of optical coherence tomography-dependent modifications in each device.

After satisfactory angiographic results, the total rate of required intervention for optical coherence tomography detected complications was significantly higher in the bioresorbable scaffolds arm compared to drug-eluting stents $\operatorname{arm}(47.8 \%$ versus $32.9 \%$, respectively; $p=0.019)$. The additional modifications encompassed further optimization in the case of device underexpansion or struts malapposition, and even stenting in the case of strut fractures, or significant edge dissection.

Conclusions: Compared to drug-eluting stents, Absord scaffold was associated with a significantly higher rate of optical coherence tomography-identified intraprocedural complications necessitating further modifications. The study provides some hints on the reasons of scaffolds failure in current $\mathrm{PCl}$ practice; it offers a new insight for the enhancement of BRS safety and presents and adds to the growing literature for successful BRS utilization.
\end{abstract}

Keywords: Bioresorbable scaffold, Optical coherence tomography, Malapposition, Strut fracture

\section{Background}

Despite significant advances in drug-eluting stents (DES) technology, metallic stents are still associated with some drawbacks, as prolonged contact with either the metallic alloy or the polymer accelerates neoatherogenesis with increased hazard of thrombosis and revascularization in

\footnotetext{
* Correspondence: Nagwaabdelrahman@aun.edu.eg

'Cardiovascular Department, Al Qassimi Hospital, Sharjah, United Arab Emirates

${ }^{2}$ Cardiovascular Department, Faculty of Medicine, Assiut University, Asyut,
} Egypt

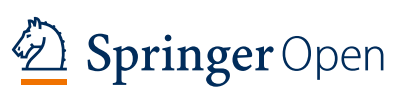

(C) The Author(s). 2020 Open Access This article is licensed under a Creative Commons Attribution 4.0 International License, which permits use, sharing, adaptation, distribution and reproduction in any medium or format, as long as you give appropriate credit to the original author(s) and the source, provide a link to the Creative Commons licence, and indicate if changes were made. The images or other third party material in this article are included in the article's Creative Commons licence, unless indicated otherwise in a credit line to the material. If material is not included in the article's Creative Commons licence and your intended use is not permitted by statutory regulation or exceeds the permitted use, you will need to obtain permission directly from the copyright holder. To view a copy of this licence, visit http://creativecommons.org/licenses/by/4.0/.

addition to vasomotion impairment and physiological blood flow disturbance $[9,11]$.

Bioresorbable vascular scaffold (BRS) development was a key step in interventional cardiology due to the added advantage of complete scaffold biodegradation, allowing the recovery of vascular pulsatility, vasomotion, and endothelial function compared to DES. The most frequently studied and clinically used scaffold is the Absorb BRS (Abbott Vascular, Santa Clara, California) [8].

Recently, interventional cardiology is at critical crossroads due to the dissatisfactions with the Absorb scaffold. The 3-year follow-up of the ABSORB III trial 
revealed a higher risk of scaffold thrombosis (ScT). A subsequent systematic review and meta-analysis with > 10,000 patients displayed doubling the risk of ScT $[5,6]$.

Owing to the inherent differences in recoil characteristics between BRS and DES and the thicker BRS struts, scaffold underexpansion and malapposition are more frequent and are possibly incriminated for the increased risk of scaffold thrombosis [2].

Optical coherence tomography (OCT) showed a great ability to identify adverse features during percutaneous coronary intervention (PCI) with DES, resulting in better clinical outcomes [13].

Recently, the 2018 ESC/EACTS Guidelines on myocardial revascularization upgraded the OCT role due to its accuracy in detecting intraprocedural complications such as malapposition, underexpansion, and edge dissection [12].

In the present study, we aimed to assess the influence of OCT on intraprocedural decision-making during implantation of Absord BRS versus everolimus-DES.

\section{Methods}

\section{Study design and population}

We studied retrospectively consecutive patients (223 patients) who had OCT-guided implantation of either Absorb BRS or everolimus-DES from March 2013 to October 2017 at Al Qassimi Hospital, Sharjah, UAE.

All patients (age $\geq 18$ years) received one or more of either Absorb BRS or everolimus-DES, either the Xience everolimus-eluting cobalt-chromium stent (Abbott Vascular, Santa Clara, USA) or the Promus EverolimusEluting Platinum-Chromium Stent (Boston Scientific, Massachusetts, USA). Patients who had OCT images with considerable artifacts were excluded from the study.

The influence of OCT guidance on intraoperative decision-making during implantation of BRS versus DES was reported by analyzing the total rate of OCT-based modification in each device.

\section{BRS implantation in 2013-2017}

All patients signed informed consent for scaffold deployment and OCT use before starting the procedure.

All patients were pretreated with aspirin $300 \mathrm{mg}$ and either clopidogrel $600 \mathrm{mg}$ or ticagrelor $180 \mathrm{mg}$ as required.

Lesion predilatation was performed upon operators' discretion in 231 (94.3\%) scaffolds at baseline. Scaffolds were implanted according to common practice with progressive inflation. Post-dilatation with non-compliant (NC) balloon was performed in 235 (96\%) scaffolds.

Planned overlapping-BRS strategy was performed when the lesion length exceeded the maximum scaffold length $(28 \mathrm{~mm})$, while unplanned overlap was done in the case of significant edge dissection or residual lesion post-scaffold deployment.

\section{Data collection schedule}

An identification number was generated for each patient in an electronic worksheet. Patient's data (demographics, biological parameters) and coronary angiography review including procedure and lesion description according to the ACC/AHA classification were recorded.

Each stent/scaffold was reviewed to detect if additional intervention was required based on the OCT analysis after what was supposed to be an angiographically successful implantation.

\section{OCT performance and analysis}

OCT acquisition was done using either the ILUMIEN OPTIS PCI Optimization System with the DragonflyDuo imaging catheter (both St. Jude Medical, MN, USA) or the LUNAWAVE OCT System with the Fastview imaging catheter (both Terumo, Japan). A non-occlusive technique with injection of iso-osmolar iodixanol (Visipaque) was used for acquisition to limit blood artifacts.

Underexpansion was defined as a minimum device area $<80 \%$ of the mean proximal and distal reference lumen area [2]. Edge dissection was defined as luminal surface disruption at the stent edge resulting in a flap (Fig. 1a). Residual disease was considered in the case of $\geq 50 \%$ narrowing of the mean luminal area in the OCT images within $5 \mathrm{~mm}$ proximal or distal to the stent/scaffold edges. Stent fracture was assumed in the case of overriding contiguous struts, disconnection from the expected device circularity, and isolated struts lying unapposed in the lumen (Fig. 1c, e). BRS strut malapposition was defined as the presence of struts separated from the adjacent vessel wall (Fig. 1g, h). In the case of DES inducing posterior dropout, malapposition was considered when the axial distance between the strut's surface and the luminal surface exceeds the strut thickness (90 um) (Fig. 1i, j) [10].

\section{Study endpoint}

The study endpoint was the total rate of required modifications based on OCT findings, including further postdilation for underexpansion and malapposition, and additional BRS/DES implantation in the case of significant edge dissection or strut fracture.

\section{Statistical analysis}

Data analysis was done using SPSS version 19 (Statistical Package for Social Science). Data were presented as number, percentage, mean, median, and standard deviation. Chi-square test and Fisher's exact test were used to compare qualitative variables. Mann-Whitney test was used to compare quantitative variables in case of non- 


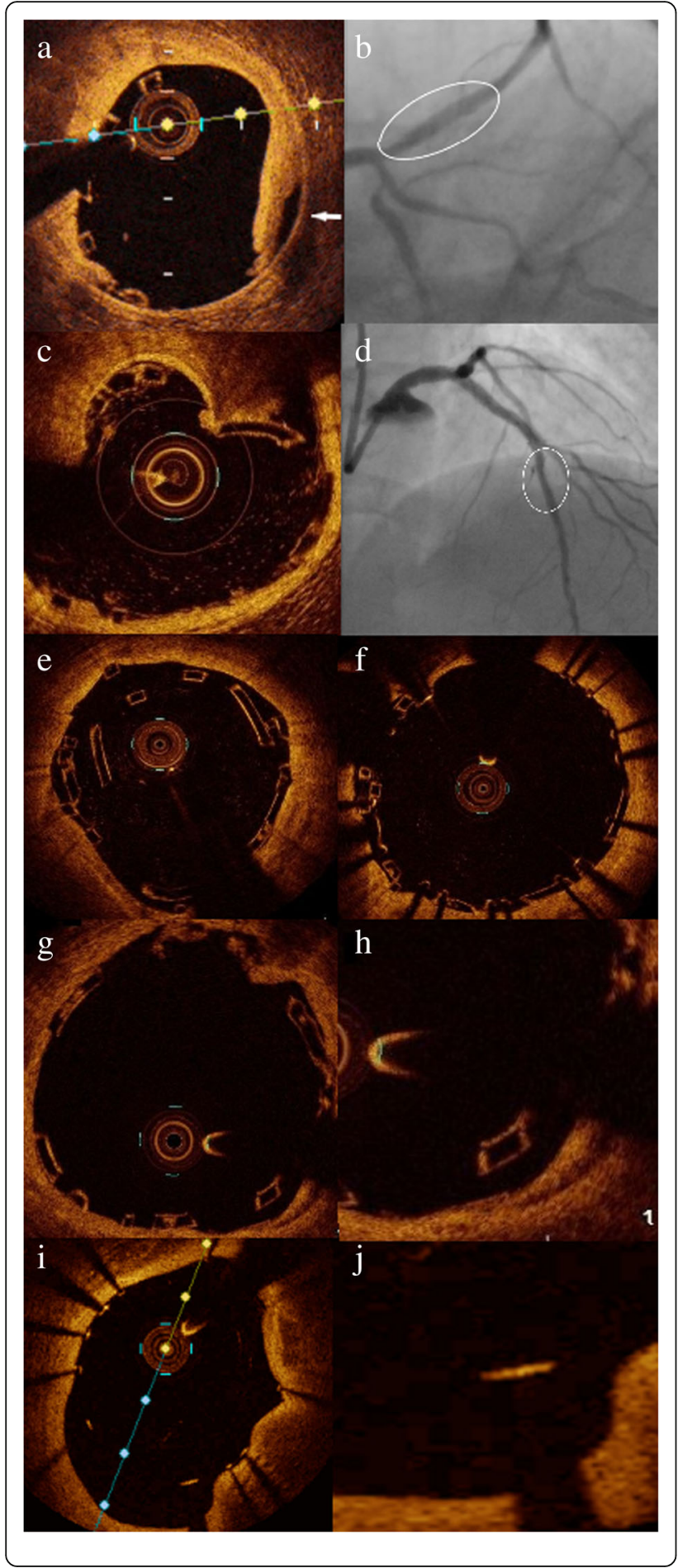

Fig. 1 OCT detected BRS and DES complications. a OCT image of intimal dissection at the edge of Absorb scaffold (arrow), b PA caudal view of the same scaffold implanted in proximal LAD (oval shape) in which edge dissection could not be identified, c OCT image of calcium spike inducing scaffold fracture with loss of the expected scaffold circularity, $\mathbf{d}$ PA cranial view of the same scaffold implanted in mid LAD (oval shape) without evidence of fracture, $\mathbf{e}$ OCT image of scaffold fracture in the form of overriding contiguous struts post culotte bifurcation stenting, $\mathbf{f}$ post successful stenting of the same scaffold using DES, $\mathbf{g}$ OCT showing malapposed scaffold struts, $\mathbf{h}$ magnified picture showing scaffold struts clearly separated from the vessel wall, i OCT picture showing metallic struts malapposition with posterior dropout, and $\mathbf{j}$ magnified picture showing that the axial distance between the strut's surface and the luminal surface exceeds the strut thickness in the malapposing struts

parametric data. A univariate regression analysis was used to identify the independent predictors of the requirement for OCT-based modifications in each arm and to estimate odds ratio (OR) and 95\% confidence intervals (CIs). A multivariate logistic analysis was used to identify the independent predictors of OCT-induced PCI modifications in all studied population. First, the following covariates were screened in univariate models: (1) clinical presentation, (2) angiographic variables (target vessel, lesion complexity), and (3) procedural variables (stent type, scaffold/stent diameter). Second, a multivariable analysis of predictors at $p<0.20$ by univariate analysis was performed to identify independent predictors of OCT-induced PCI modifications and to estimate adjusted odds ratios and 95\% confidence intervals (CIs). $p$ value $<0.05$.was considered statistically significant.

\section{Results}

Patients and procedures

Consecutive patients $(n: 242)$ post OCT-guided implantation of either Absorb BRS or everolimus-DES from March 2013 to October 2017 were assessed for eligibility. Nineteen patients were excluded because of artifacts rendering proper OCT images analysis unfeasible. We included 223 patients, with either Absorb BRS (162 patients; $75 \%$ males; mean age $53.5 \pm 26$ years) or everolimus-DES (61 patients; 77\% males; mean age $51 \pm$ 25 years).

The Absorb arm had 179 lesions (245 scaffolds), versus 65 lesions (82 stents) in the DES arm.

Patients' baseline characteristics and PCI indications were almost similar in both groups (Table 1). Lesion and procedural characteristics are summarized in Table 2.

\section{OCT-detected intraoperative complications}

After satisfactory angiographic results, the total rate of required intervention for OCT-detected complications was significantly higher in the BRS arm compared to the 
Table 1 Baseline demographics and clinical characteristics

\begin{tabular}{|c|c|c|c|}
\hline \multirow[t]{2}{*}{ Characteristic } & \multirow{2}{*}{$\begin{array}{l}\text { Absorb scaffold }(N=162) \\
\text { No. }(\%)\end{array}$} & \multirow{2}{*}{$\begin{array}{l}\text { Everolimus-DES }(N=61) \\
\text { No. }(\%)\end{array}$} & \multirow[t]{2}{*}{$p$ value } \\
\hline & & & \\
\hline Male & $122(75.3 \%)$ & $47(77.0 \%)$ & 0.787 \\
\hline Age/years & $54.36 \pm 10.02$ & $54.13 \pm 10.89$ & 0.805 \\
\hline Body mass index & $28.93 \pm 4.17$ & $28.97 \pm 5.32$ & 0.361 \\
\hline Hypertension & $104(64.2 \%)$ & $35(57.4 \%)$ & 0.349 \\
\hline Diabetes mellitus & $93(57.4 \%)$ & $41(67.2 \%)$ & 0.183 \\
\hline Insulin-treated & $29(17.9 \%)$ & $15(24.6 \%)$ & 0.263 \\
\hline Dyslipidemia & $120(74.1 \%)$ & $46(75.4 \%)$ & 0.838 \\
\hline Current smoker & $57(36.5 \%)$ & $23(37.7 \%)$ & 0.873 \\
\hline Previous myocardial infarction & $25(15.4 \%)$ & $10(16.4 \%)$ & 0.860 \\
\hline Post-CABG & $2(1.2 \%)$ & $3(4.9 \%)$ & 0.127 \\
\hline \multicolumn{4}{|l|}{ Clinical presentation: } \\
\hline Silent ischemia & 19(11.7\%) & $7(11.5 \%)$ & 0.958 \\
\hline Stable angina & $50(30.9 \%)$ & $21(34.4 \%)$ & 0.611 \\
\hline Unstable angina/NSTEMI & $63(38.9 \%)$ & $28(45.9 \%)$ & 0.928 \\
\hline Primary $\mathrm{PCl}$ & $20(12.3 \%)$ & $4(6.6 \%)$ & 0.214 \\
\hline Late or lysed STEMI & $10(6.2 \%)$ & $1(1.6 \%)$ & 0.297 \\
\hline
\end{tabular}

Plus-minus values are means $\pm S D$

$B M I$ body mass index is the weight in kilograms divided by the square of the height in meters, $C A B G$ coronary artery bypass grafting, $D E S$ drug-eluting stents, $P C I$ percutaneous coronary intervention, STEMI ST-elevation myocardial infarction

DES arm (47.8\% versus $32.9 \%$, respectively; $p=0.019)$, (Table 3).

$B R S$ bioresorbable scaffolds, DES drug-eluting stent

Stenting for OCT-detected dissection was required in $6.5 \%$ of BRS and $1.2 \%$ of DES arms $(p=0.06)$ (Fig. 1a, b), (video 1 and 2 in the supplemental material). Underexpansion necessitating stenting was observed in 2 scaffolds $(0.8 \%)$ that showed significant underexpansion despite multiple attempts of postdilatation with $\mathrm{NC}$ balloon at high pressure, so upgrade of the NC balloon size and pressure beyond the manufacturer limit was required, and thus stents were implanted for fear of struts fracture. Stenting due to fractured struts was required in 4 scaffolds (1.6\%), but not in the DES arm (Fig. 1c-f). Thrombus aspiration post BRS implantation was attempted by the operators in a case where thrombus was extending to more than 3 frames $(6 \mathrm{~mm})$, appearing as a thrombus protruding between struts in one frame $(8 \times 6 \mathrm{~mm})$, and as small thrombi moving freely inside the lumen in other frames. Thrombus stenting was done in a case of a large thrombus $(4 \times 3 \mathrm{~mm})$ protruding out of the distal scaffold edge with a free tail in the vessel lumen, which could be a substrate for scaffold thrombosis, or at least microvascular occlusion and no-reflow. The total rate of bailout devices used was significantly higher in the BRS arm $(n: 25 ; 10.2 \%)$ compared to DES $\operatorname{arm}(n: 1 ; 1.2 \%)(p=0.009)$.

Among 6 cases with acute scaffold failure, two were due to heavily calcified lesions with subsequent underexpansion, as well as OCT-detected struts fracture in 4 scaffolds. All 6 lesions were treated successfully with DES (Fig. 1c-f). There were no adverse events associated with OCT imaging in the studied population.

NSTEMI presentation, type B2/C lesion, moderate/severe calcification, and overlapping stents, all were found to have a significant positive relationship with the need for intraoperative modification post-OCT review in the BRS arm. Bifurcation stenting was the only variable that has a positive relation in the DES arm (Table 4).

By performing a multivariate logistic regression analysis of all studied population, BRS scaffold, type B2/C lesion, moderate/severe calcification, and primary $\mathrm{PCI}$ presentation, all were found to have a significant relationship with the OCT-detected complications post device implantation (Table 5).

\section{Discussion}

The invention of bioresorbable vascular scaffolds was considered a revolution in the field of interventional cardiology due to the potential advantages of scaffold bioresorption.

To our knowledge, this is the first study to compare the influence of OCT on the operators' decisions during deployment of Absorb BRS versus everolimus-eluted metallic stents.

Optimal scaffold placement entails a balance between perfect struts embedment and absence of edge dissection. Uncorrected residual stenosis, struts malapposition, 
Table 2 Lesions and procedural characteristics

\begin{tabular}{|c|c|c|c|}
\hline \multirow[t]{2}{*}{ Variable } & \multicolumn{3}{|l|}{ Lesion based } \\
\hline & Absorb scaffold $(N=179)$ & Everolimus-DES $(N=65)$ & $p$ value \\
\hline \multicolumn{4}{|l|}{ Target vessel: } \\
\hline LM & $4(2.2 \%)$ & $15(23.1 \%)$ & $<0.001$ \\
\hline LAD & $100(55.9 \%)$ & $40(61.5 \%)$ & 0.428 \\
\hline LCX & $35(19.6 \%)$ & $12(18.4 \%)$ & 0.863 \\
\hline RCA & $43(24.0 \%)$ & $10(15.4 \%)$ & 0.148 \\
\hline SVG & $1(0.6 \%)$ & $1(1.5 \%)$ & 0.463 \\
\hline \multirow[t]{3}{*}{$\mathrm{ACC} / \mathrm{AHA}$ lesion class B2/C } & $116(64.8 \%)$ & $46(70.8 \%)$ & 0.383 \\
\hline & \multicolumn{3}{|l|}{ Scaffold/stent based } \\
\hline & Absorb scaffold $(n=245)$ & Everolimus-DES ( $n=82$ ) & $p$ value \\
\hline Bifurcation stenting & $18(7.3 \%)$ & 17 (20.7\%) & $<0.001$ \\
\hline Moderate/severe calcification & $86(35.1 \%)$ & $38(46.3 \%)$ & 0.069 \\
\hline Thrombus & $24(9.8 \%)$ & $5(6.1 \%)$ & 0.308 \\
\hline Overlap & $141(57.6 \%)$ & $59(72.0 \%)$ & 0.020 \\
\hline In-stent implantation & $15(6.1 \%)$ & $28(34.1 \%)$ & $<0.001$ \\
\hline Chronic total occlusion & $11(4.5 \%)$ & $3(3.7 \%)$ & 0.74 \\
\hline Pre-dilatation & $231(94.3 \%)$ & $71(86.6 \%)$ & 0.023 \\
\hline Pre-dilatation NC-balloon & $161(65.7 \%)$ & $37(45.1 \%)$ & 0.001 \\
\hline Pre-dilatation cutting balloon & $24(9.8 \%)$ & $19(23.2 \%)$ & 0.002 \\
\hline Pre-dilatation balloon/device diameter-ratio & $0.96 \pm 0.11$ & $0.90 \pm 0.13$ & $<0.001$ \\
\hline Pre-dilatation inflation pressure/atm & $14.38 \pm 3.92$ & $13.79 \pm 3.64$ & 0.464 \\
\hline Stent inflation pressure/atm & $11.95 \pm 2.67$ & $12.70 \pm 2.87$ & 0.099 \\
\hline Stent inflation time/sec & $76.66 \pm 18.00$ & $16.50 \pm 5.57$ & $<0.001$ \\
\hline Post-dilatation NC-balloon & 235(95.9\%) & $81(98.8 \%)$ & 0.303 \\
\hline Post-dilatation balloon/device diameter ratio & $1.10 \pm 0.09$ & $1.08 \pm 0.12$ & 0.025 \\
\hline Post-dilatation balloon-device diameter/mm & $0.34 \pm 0.25$ & $0.29 \pm 0.34$ & 0.04 \\
\hline Post-dilatation inflation pressure/atm & $17.89 \pm 4.04$ & $16.84 \pm 4.03$ & 0.028 \\
\hline Post-dilatation inflation time/sec & $15.88 \pm 6.22$ & $13.70 \pm 3.44$ & 0.004 \\
\hline Device success & $239(97.6 \%)$ & $81(98.8 \%)$ & 0.685 \\
\hline Use of bailout device & $25(10.2 \%)$ & $1(1.2 \%)$ & 0.009 \\
\hline
\end{tabular}

Table 3 Comparison of OCT-guided modification in BRS vs. DES

\begin{tabular}{llll}
\hline Modifications & BRS $(\boldsymbol{n}=\mathbf{2 4 5})$ & DES $(\boldsymbol{n}=\mathbf{8 2})$ & No. (\%) \\
\hline Total modifications & No. (\%) & \multicolumn{1}{c}{$\begin{array}{l}(32.9) \\
\text { value }\end{array}$} \\
Post-dilatation due to underexpansion or malapposition & $117(47.6)$ & $26(31.7)$ & 0.019 \\
Dissection stenting & $90(36.7)$ & $1(1.2)$ & 0.410 \\
Stenting due to underexpansion & $16(6.5)$ & $0(0.0)$ & 0.060 \\
Fracture stenting & $2(0.8)$ & $0(0.0)$ & 0.411 \\
Thrombus aspiration & $4(1.6)$ & $0(0.0)$ & 0.576 \\
Thrombus stenting & $1(0.4)$ & $0(0.0)$ & 0.562 \\
Residual lesion stenting & $1(0.4)$ & $0(0.0)$ & 0.562 \\
\hline
\end{tabular}


Table 4 Univariate logistic regression analysis of OCT based modification in BRS and DES

\begin{tabular}{|c|c|c|c|c|}
\hline & \multicolumn{2}{|l|}{ BRS } & \multicolumn{2}{|l|}{ DES } \\
\hline & Odds ratio $(95 \% \mathrm{Cl})$ & $p$ value & Odds ratio $(95 \% \mathrm{Cl})$ & $p$ value \\
\hline \multicolumn{5}{|l|}{ Clinical presentation: } \\
\hline CSA & $1.77(0.9-3.48)$ & 0.095 & $1.02(0.34-3.04)$ & 0.964 \\
\hline UA & $1.34(0.54-3.31)$ & 0.519 & $0.99(0.21-4.59)$ & 0.99 \\
\hline NSTEMI & $2.165(1.03-4.52)$ & $0.04^{*}$ & $0.42(0.13-1.39)$ & 0.158 \\
\hline Late/lysed MI & $0.7(1.19-2.59)$ & 0.59 & - & - \\
\hline Primary $\mathrm{PCl}$ & $2.2(0.82-5.84)$ & 0.113 & $1.71(0.22-13.08)$ & 0.603 \\
\hline Silent ischemia & $0.45(0.16-1.26)$ & 0.131 & $5.0(0.88-28.33)$ & 0.069 \\
\hline \multicolumn{5}{|l|}{ Target vessel: } \\
\hline$\angle A D$ & $1.03(0.57-1.87)$ & 0.911 & $2.18(0.7-6.72)$ & 0.175 \\
\hline LCX & $0.56(0.25-1.21)$ & 0.144 & $0.76(0.172-3.38)$ & 0.721 \\
\hline RCA & $1.62(0.815-3.24)$ & 0.168 & $0.63(0.14-2.72)$ & 0.539 \\
\hline ACC/AHA B2/C & $1.82(1.016-3.28)$ & $0.044^{*}$ & $1.96(0.63-6.07)$ & 0.239 \\
\hline Bifurcation stenting & $1.5(0.57-3.95)$ & 0.407 & $2.93(0.981-8.79)$ & $0.05^{*}$ \\
\hline Moderate/severe calcification & $2.41(1.407-4.15)$ & $0.001^{*}$ & $1.30(0.55-3.5)$ & 0.484 \\
\hline Overlap & $1.84(1.09-3.08)$ & $0.021^{*}$ & $1.56(0.53-4.57)$ & 0.413 \\
\hline In-stent restenosis & $1.81(0.62-5.27)$ & 0.272 & $1.9(0.74-5.07)$ & 0.171 \\
\hline Сто & $0.65(0.18-2.29)$ & 0.509 & $1.01(0.08-11.76)$ & 0.988 \\
\hline Ostial & $0.65(0.32-1.31)$ & 0.234 & $1.39(0.55-3.52)$ & 0.484 \\
\hline
\end{tabular}

ACC/AHA American College of Cardiology/American Heart Association, BRS bioresorbable scaffold, CSA chronic stable angina, Cl confidence interval, CTO chronic total occlusion, DES drug-eluting stents, LAD left anterior descending, LCX left circumflex, NSTEMI non-ST elevation myocardial infarction, OCT optical coherence tomography, $P C I$ percutaneous coronary intervention, $R C A$ right coronary artery, UA unstable angina

and edge dissections increase the risk of future scaffold restenosis and thrombosis [2].

In the present study, there was a significant difference in the impact of OCT on intraoperative decision-making during implantation of BRS versus DES. Despite angiographic success, we reported a significantly higher rate of OCT detected device-related complications in the BRS arm (47.8\%) compared to the DES arm (32.9\%).

The detected complications necessitated further modifications such as further optimization in case of device underexpansion or struts malapposition, and even additional stenting in case of strut fractures, or significant edge dissection reaching up to $10.2 \%$ of bailout device use in the BRS arm versus $1.2 \%$ only in the DES arm.

In ABSORB cohort B substudy, OCT detected suboptimal deployment in more than $25 \%$ of patients with mainly type A lesions, and this number might have theoretically increased with the expanded BRS use into complex lesions $[1,14]$. This theory was verified by our findings, where type $\mathrm{B} 2 / \mathrm{C}$ lesion, moderate/severe lesion calcification, and overlapping stents had a positive relationship with the need for OCT-guided additional interventions, emphasizing the importance of lesion selection before BRS implantation. BRS scaffold was found to have a significant positive relationship with the OCT-based intraoperative modification, thus highlighting the value of OCT guidance for BRS optimization.

Even if not identified on angiography, the OCTdetected complications may have a critical impact on patients' outcome if left untreated [13]. It is well known that the recent Absorb setback was mainly due to a higher tendency of very late scaffold thrombosis in the ABSORB trials $[5,15]$.

Increased rates of subacute scaffold thrombosis in ABSORB III trial were explained by the high rate of residual stenosis, while the high very late scaffold thrombosis rate in ABSORB II was attributed to underexpansion and incomplete coverage due to malapposition, as detected with intravascular ultrasound (IVUS) [3, 4]. In the ABSORB Japan trial, OCT showed incomplete coverage due to overhanging struts in very late scaffold thrombosis cases as malapposing struts prevent scaffold bioresorption and delay endothelialization, thus increasing the risk of thrombosis [7].

The difference in the rate of required interventions between both devices can be explained by the intrinsic biomechanical differences due to the eccentric expansion pattern of the Absorb scaffold that results into a higher rate of underexpansion and struts malapposition [2]. Moreover, the radiolucency of the scaffold to the conventional angiography renders underexpansion and fractures less likely to be spotted by this modality. 
Table 5 Multivariate logistic regression analysis of OCT-based modification in the studied population

\begin{tabular}{|c|c|c|c|c|}
\hline & \multicolumn{2}{|l|}{ Univariate } & \multicolumn{2}{|l|}{ Multivariate } \\
\hline & Odds ratio $(95 \% \mathrm{Cl})$ & $p$ value & Odds ratio $(95 \% \mathrm{Cl})$ & $p$ value \\
\hline Stent type (BRS) & $1.74(1.03-2.94)$ & $0.038^{*}$ & $2.35(1.28-4.31)$ & $0.006^{*}$ \\
\hline \multicolumn{5}{|l|}{ Clinical presentation: } \\
\hline CSA & $0.76(0.48-1.22)$ & 0.271 & - & - \\
\hline UA & $0.79(0.42-1.47)$ & 0.459 & - & - \\
\hline NSTEMI & $2.02(1.19-3.42)$ & $0.009^{*}$ & $1.71(0.97-3.01)$ & 0.062 \\
\hline Late/Lysed Ml & $1.8(0.67-4.82)$ & 0.238 & - & - \\
\hline Primary $\mathrm{PCl}$ & $2.51(1.15-5.46)$ & $0.02^{*}$ & $2.55(1.1-5.9)$ & $0.028^{*}$ \\
\hline Silent ischemia & $0.96(0.49-1.89)$ & 0.917 & - & - \\
\hline \multicolumn{5}{|l|}{ Target vessel: } \\
\hline LM & $0.52(0.2-1.34)$ & 0.177 & $0.6(0.18-1.93)$ & 0.397 \\
\hline LAD & $0.87(0.56-1.37)$ & 0.57 & - & - \\
\hline LCX & $1.85(0.96-3.55)$ & 0.064 & $1.34(0.64-2.82)$ & 0.431 \\
\hline RCA & $0.67(0.41-1.11)$ & 0.124 & $0.74(0.41-1.34)$ & 0.327 \\
\hline ACC/AHA B2/ C lesion class & $1.84(1.1-3.09)$ & $0.02^{*}$ & $1.78(1.02-3.11)$ & $0.039^{*}$ \\
\hline Bifurcation stenting & $0.59(0.29-1.2)$ & 0.15 & $0.52(0.22-1.21)$ & 0.131 \\
\hline Moderate/severe calcification & $1.88(1.19-2.95)$ & $0.006^{*}$ & $1.99(1.2-3.3)$ & $0.029^{*}$ \\
\hline Overlap & $1.64(1.04-2.6)$ & $0.03^{*}$ & $1.39(0.82-2.33)$ & 0.214 \\
\hline In-stent restenosis & $0.75(0.39-1.43)$ & 0.39 & - & - \\
\hline Сто & $1.36(0.44-4.16)$ & 0.585 & - & - \\
\hline Ostial lesion & $1.34(0.79-2.28)$ & 0.275 & - & - \\
\hline Stent diameter & $1.09(0.68-1.75)$ & 0.714 & - & - \\
\hline
\end{tabular}

ACC/AHA American College of Cardiology/American Heart Association, BRS bioresorbable scaffold, CSA chronic stable angina, CI confidence interval, CTO chronic total occlusion, $L A D$ left anterior descending, LCX left circumflex, NSTEMI non-ST elevation myocardial infarction, OCT optical coherence tomography, PCI percutaneous coronary intervention, RCA right coronary artery, UA unstable angina

A significant difference in the implantation technique adopted by the operators during BRS versus DES deployment was noted, as post-dilatation balloon/device diameter ratio, inflation time, and pressure were higher in the BRS $\operatorname{arm}(1.10 \pm 0.09$ vs $1.08 \pm 0.12, p=0.025 ; 15.88 \pm 6.22 \mathrm{~s}$ vs13.70 $\pm 3.44 \mathrm{~s}, p=0.004$; and $17.89 \pm 4.04 \mathrm{~atm}$ vs $16.84 \pm$ $4.03 \mathrm{~atm}, p=0.028$, respectively), most likely governed by OCT findings of malapposition and underexpansion.

OCT displayed intraoperative complications requiring further interventions in $32.9 \%$ of DES arm, in concordance with the CLI-OPCI study that reported a $34.7 \%$ rate of additional interventions based on OCT findings during PCI using metallic stents [13]. The current results highlight the value of OCT-guidance also during DES implantation.

\section{Study limitations}

The study has some limitations, including those typical of non-randomized studies; however, the retrospective design has some advantages in the present study, as it prevented the operators' bias while studying the impact of OCT on intraprocedural operators' behavior. Additionally, substantial discrepancies between groups could not be avoided due to the retrospective time-limited study design; nevertheless, despite higher lesions complexity in the DES group, a significantly lower rate of modifications was required and thus strengthens the study results; however, we should not forget that no method can accurately adjust for all known and unknown confounders. Besides, any malapposition not considering axial and/or longitudinal distance (axial distance $>300 \mu \mathrm{m}$ and longitudinal extension $>1.0 \mathrm{~mm}$, not associated with side branches) may lead to the overestimation of complications detected by OCT after device implantation because minor malapposition $(<0.35 \mathrm{~mm})$ will be resolved with neointima at follow-up. Finally, despite that the intraprocedural OCT assessment was verified by a different team during the study, the lack of corelab analysis is considered a limitation.

\section{Conclusions}

Compared to DES, Absorb scaffold was associated with a significant higher rate of OCT-detected intraprocedural complications requiring further modifications. The study provides some hints on the reasons of scaffolds failure in current PCI practice; it offers a new insight for 
the enhancement of BRS safety and presents and adds to the growing literature for successful BRS utilization.

\section{Supplementary Information}

The online version contains supplementary material available at https://doi. org/10.1186/s43044-020-00110-z

Additional file 1: Video 1. Coronary angiography of PA caudal view showing ABSORB scaffold implanted in proximal LAD segment, without evidence of proximal edge dissection, linked to Fig. $1 \mathrm{~b}$.

Additional file 2: Video 2. OCT run in proximal segment of same scaffold in proximal $L A D$, showing proximal edge dissection as evident by intimal disruption at the scaffold edge, linked to Fig. 1a.

\section{Abbreviations}

BRS: Bioresorbable scaffold(s); DES: Drug-eluting stent(s); MI: Myocardial infarction; NC balloon: Non-compliant balloon; OCT: Optical coherence tomography; PCl: Percutaneous coronary intervention; ScT: Scaffold thrombosis; STEMI: ST-segment elevation myocardial infarction

\section{Acknowledgements}

Not applicable

\section{Authors' contributions}

All authors have made an important contribution to the study and are thoroughly familiar with the primary data. All authors are responsible for the contents and have read and approved the manuscript. Administrative support: AA. Provision of study materials or patients: NA, AA. Collection and assembly of data: NA. Conception and design, data analysis and interpretation, manuscript writing, and final approval of manuscript: NA, AA, $\mathrm{HH}, \mathrm{YK}$, and $\mathrm{AH}$.

\section{Funding}

This research did not receive any specific grant from any funding agencies.

\section{Availability of data and materials}

Raw data will be available upon request by the editorial board

\section{Ethics approval and consent to participate}

The protocol complied with Helsinki declaration and was approved by both Sharjah medical district research ethics committee, IRB: MOHAP/SHJ/4I2017, and Assiut University Hospitals (homeland of the co-authors), IRB: 17200359. Due to the retrospective nature of the study, waiver of the informed consent was agreed upon provided confidentiality of the patients' identities.

\section{Consent for publication}

Due to the retrospective nature of the study, waiver of the informed consent was agreed upon provided confidentiality of the patients' identities

\section{Competing interests}

The authors have no conflicts of interest to declare.

Received: 18 June 2020 Accepted: 14 October 2020

Published online: 05 November 2020

\section{References}

1. Al Nooryani A, Elabbassi WN, AlBaba M et al (2019) Long term outcome of first 300 implanted Absorb vascular bioresorbable vascular scaffolds in an allcomers Middle East population. J Int Med Res 47:173-187

2. Allahwala UK, Cockburn JA, Shaw E, Figtree GA, Hansen PS, Bhindi R (2015) Clinical utility of optical coherence tomography (OCT) in the optimisation of Absorb bioresorbable vascular scaffold deployment during percutaneous coronary intervention. Eurolntervention 10:1154-1159

3. Chevalier B, Cequier A, Dudek D et al (2018) Four-year Follow-up of the randomised comparison between an everolimus-eluting bioresorbable scaffold and an everolimus-eluting metallic stents for the treatment for coronary artery stenosis, ABSORB I| trial. Eurolntervention 13:1561-1564. https://doi.org/10.4244/EIJ-D-17-00873
4. Ellis SG, Kereiakes DJ, Metzger DC et al (2015) Everolimus-Eluting Bioresorbable Scaffolds for Coronary Artery Disease. N Engl J Med 373:19051915

5. Forrestal BJ, Lipinski MJ. Bioresorbable scaffolds: fading away or hope for the future? J Am Coll Cardiol Feb 07, 2018. https://www.acc.org/latest-incardiology/articles/2018/02/07/07/45/bioresorbable-scaffolds.

6. Kereiakes DJ, Ellis SG, Metzger C et al for the ABSORB III Investigators. Threeyear clinical outcomes with everolimus-eluting bioresorbable scaffolds: results from the randomized ABSORB III Trial. J Am Coll Cardiol, Volume 70, Issue 23, December 2017:2852-2862.

7. Ken Kozuma. ABSORB Japan Results: 3-year clinical and angiographic. Presented at: the Euro PCR, May 17, 2017, Paris, France

8. Lipinski MJ, Escarcega RO, Lhermusier T, Waksman R (2014) The effects of novel, bioresorbable scaffolds on coronary vascular pathophysiology. J Cardiovasc Transl Res 7:413-425

9. Lüscher TF, Steffel J, Eberli FR et al (2007) Drug-eluting stent and coronary thrombosis. Biological mechanisms and clinical implications. Circulation 115 1051-1058

10. Mattesini A, Secco G, Dall'Ara G et al (2014) ABSORB biodegradable stents versus second-generation metal stents, a comparison study of 100 complex lesions treated under OCT guidance. J Am Coll Cardiol Intv 7:1936-8798

11. Nakazawa G, Otsuka F, Nakano M et al (2011) The pathology of neoatherosclerosis in human coronary implants bare-metal and drugeluting stents. J Am Coll Cardiol 57:1314-1322

12. Neumann FJ, Sousa-Uva M, Ahlsson A et al (2018) 2018 ESC/EACTS Guidelines on myocardial revascularization. Eur Heart J 00:1-96. https://doi. org/10.1093/eurheartj/ehy394

13. Prati F, Di Vito L, Biondi-Zoccai G et al (2012) Angiography alone versus angiography plus optical coherence tomography to guide decision-making during percutaneous coronary intervention: the Centro per la Lotta contro I'Infarto-Optimisation of Percutaneous Coronary Intervention (CLI-OPCl) study. Eurolntervention. 8:823-829

14. Serruys PW, Onuma Y, Dudek D et al (2011) Evaluation of the second generation of a bioresorbable everolimus-eluting vascular scaffold for the treatment of de novo coronary artery stenosis: 12-month clinical and imaging outcomes. J Am Coll Cardiol 58:1578-1588

15. Serruys PW, Chevalier B on behalf of the ABSORB I| Investigators. ABSORB II: a prospective, randomized trial of an everolimus-eluting bioresorbable scaffold versus an everolimus-eluting metallic stent in patients with coronary artery disease. Presented at: Transcatheter Cardiovascular Therapeutics conference, Sep13,2014, Washington-DC, USA

\section{Publisher's Note}

Springer Nature remains neutral with regard to jurisdictional claims in published maps and institutional affiliations.

\section{Submit your manuscript to a SpringerOpen ${ }^{\circ}$ journal and benefit from:}

- Convenient online submission

- Rigorous peer review

- Open access: articles freely available online

- High visibility within the field

- Retaining the copyright to your article

Submit your next manuscript at $>$ springeropen.com 